\title{
Simulation study for measuring thermal conductivity and thermal diffusivity of nanofluid using hot wire-laser probe beam displacement technique
}

\begin{abstract}
In this paper, we present thermal conductivity and thermal diffusivity of chromium $(\mathrm{Cr})$ nanoparticle suspended in ethylene glycol at different volume fraction concentrations of nanoparticle. All samples have been prepared using single step method. The thermal conductivity and thermal diffusivity were measured via hot wire-laser beam deflection method. A numerical simulation of the heat conduction equation and probe beam deflection has been performed to determine the effective thermal conductivity and effective thermal diffusivity of $\mathrm{Cr}$ nanofluids. By fitting the experimental data to the numerical data, the thermal conductivity and thermal diffusivity of chromium $(\mathrm{Cr})$-ethylene glycol was obtained. The results also show that the effective thermal conductivity and thermal diffusivity of nanofluids increases with the increasing of nanoparticle volume fraction concentration in base fluid.
\end{abstract}

Keyword: Chromium nanofluid; Hot wire; Probe beam 